PROCEEDINGS OF THE AMERICAN MATHEMATICAL SOCIETY

Volume 124, Number 11, November 1996

\title{
COLORING FINITE SUBSETS OF UNCOUNTABLE SETS
}

\author{
PÉTER KOMJÁTH AND SAHARON SHELAH
}

(Communicated by Andreas R. Blass)

\begin{abstract}
It is consistent for every $1 \leq n<\omega$ that $2^{\omega}=\omega_{n}$ and there is a function $F:\left[\omega_{n}\right]^{<\omega} \rightarrow \omega$ such that every finite set can be written in at most $2^{n}-1$ ways as the union of two distinct monocolored sets. If GCH holds, for every such coloring there is a finite set that can be written at least $\frac{1}{2} \sum_{i=1}^{n}\left(\begin{array}{c}n+i \\ n\end{array}\right)\left(\begin{array}{c}n \\ i\end{array}\right)$ ways as the union of two sets with the same color.
\end{abstract}

\section{INTRODUCTION}

In [6] we proved that for every coloring $F:\left[\omega_{n}\right]^{<\omega} \rightarrow \omega$ there exists a set $A \in\left[\omega_{n}\right]^{<\omega}$ which can be written at least $2^{n}-1$ ways as $A=H_{0} \cup H_{1}$ for some $H_{0} \neq H_{1}, F\left(H_{0}\right)=F\left(H_{1}\right)$, and that for $n=1$ there is in fact a function $F$ for which this is sharp. Here we show that for every $n<\omega$ it is consistent that $2^{\omega}=\omega_{n}$ and for some function $F$ as above for every finite set $A$ there are at most $2^{n}-1$ solutions of the above equation. We use historic forcing, which was first used in [1] and [7], then in [5] and [4]. Under GCH, we improve the positive result of [6] by showing that for every $F$ as above some finite set can be written at least $T_{n}=\frac{1}{2} \sum_{i=1}^{n}\left(\begin{array}{c}n+i \\ n\end{array}\right)\left(\begin{array}{c}n \\ i\end{array}\right)$ ways as the union of two sets with the same $F$ value.

With the methods of [6] it is easy to show the following corollary of our independence result. It is consistent that $2^{\omega}=\omega_{n}$ and there is a function $f: \mathbb{R} \rightarrow \omega$ such that if $x$ is a real number then $x$ cannot be written more than $2^{n}-1$ ways as the arithmetic mean of some $y \neq z$ with $f(y)=f(z) .((y, z)$ and $(z, y)$ are not regarded as distinct.)

Notation. We use the standard set theory notation. If $S$ is a set, $\kappa$ a cardinal, then $[S]^{\kappa}=\{A \subseteq S:|A|=\kappa\},[S]^{<\kappa}=\{A \subseteq S:|A|<\kappa\},[S]^{\leq \kappa}=\{A \subseteq S:|A| \leq \kappa\}$. $P(S)$ is the power set of $S$. If $f$ is a function, $A$ a set, then $f[A]=\{f(x): x \in A\}$.

\section{THE INDEPENDENCE RESULT}

Theorem 1. For $1 \leq n<\omega$ it is consistent that $2^{\omega}=\omega_{n}$ and there is a function $F:\left[\omega_{n}\right]^{<\omega} \rightarrow \omega$ such that for every $A \in\left[\omega_{n}\right]^{<\omega}$ there are at most $2^{n}-1$ solutions of $A=H_{0} \cup H_{1}$ with $H_{0} \neq H_{1}, F\left(H_{0}\right)=F\left(H_{1}\right)$.

Received by the editors March 31, 1995 and, in revised form, May 9, 1995.

1991 Mathematics Subject Classification. Primary 03E35.

Key words and phrases. Axiomatic set theory, independence proofs, combinatorial set theory.

The first author was supported by the Hungarian OTKA, Grant No. T014105.

This paper is number 516 in the cumulative list of Shelah's papers. His research was supported by the Basic Research Foundation of the Israel Academy of Sciences and Humanities.

(c)1996 American Mathematical Society 
For $\alpha<\omega_{n}$ fix a bijection $\varphi_{\alpha}: \alpha \rightarrow|\alpha|$. For $x \in\left[\omega_{n}\right]^{<\omega}$ define $\gamma_{i}(x)$ for $i<k=\min (n,|x|)$ as follows: $\gamma_{0}(x)=\max (x)$,

$$
\gamma_{i+1}(x)=\varphi_{\gamma_{0}(x)}^{-1}\left(\gamma_{i}\left(\varphi_{\gamma_{0}(x)}\left[x \cap \gamma_{0}(x)\right]\right)\right),
$$

and $\gamma(x)=\left\{\gamma_{0}(x), \ldots, \gamma_{k-1}(x)\right\}$

So, for example, if $n=0$ then $\gamma(x)=\emptyset$, if $n=1, x \neq \emptyset$, then $\gamma(x)=\left\{\gamma_{0}(x)\right\}=$ $\{\max (x)\}$

Lemma 1. Given $s \in\left[\omega_{n}\right]^{\leq n}$, there are at most countably many $x \in\left[\omega_{n}\right]^{<\omega}$ such that $\gamma(x)=s$.

Proof. By induction on $n$.

Let $\Phi(s)=\bigcup\{x: \gamma(x) \subseteq s\}$, a countable set for $s \in\left[\omega_{n}\right]^{<\omega}$.

Definition. The two sets $x, y \in\left[\omega_{n}\right]^{<\omega}$ are isomorphic if the structures $(x ;<$, $\left.\gamma_{0}(x), \ldots, \gamma_{k-1}(x)\right),\left(y ;<, \gamma_{0}(y), \ldots, \gamma_{k-1}(y)\right)$ are isomorphic, i.e., $|x|=|y|$ and the positions of the elements $\gamma_{i}(x), \gamma_{i}(y)$ are the same.

Notice that for every finite $j$ there are just finitely many isomorphism types of $j$-element sets.

The elements of $P$, the applied notion of forcing, will be some structures of the form $p=(s, f)$ where $s \in\left[\omega_{n}\right]^{<\omega}$ and $f: P(s) \rightarrow \omega$.

The only element of $P_{0}$ is $\mathbf{1}_{P}=(\emptyset,\langle\emptyset, 0\rangle)$; it will be the largest element of $P$. The elements of $P_{1}$ are of the form $p=(\{\xi\}, f)$ where $f(\emptyset)=0 \neq f(\{\xi\})$ for $\xi<\omega_{n}$.

Given $P_{t}, p=(s, f)$ is in $P_{t+1}$ if the following is true. $s=\Delta \cup a \cup b$ is a disjoint decomposition. $p^{\prime}=\left(\Delta \cup a, f^{\prime}\right)$ and $p^{\prime \prime}=\left(\Delta \cup b, f^{\prime \prime}\right)$ are in $P_{t}$, where $f^{\prime}=f\left|P(\Delta \cup a), f^{\prime \prime}=f\right| P(\Delta \cup b)$. There is $\pi: \Delta \cup a \rightarrow \Delta \cup b$, an isomorphism between $\left(\Delta \cup a,<, P(\Delta \cup a), f^{\prime}\right)$ and $\left(\Delta \cup b,<, P(\Delta \cup b), f^{\prime \prime}\right)$. $\pi \mid \Delta$ is the identity. For $H \subseteq \Delta \cup a$ the sets $H$ and $\pi[H]$ are isomorphic. $a \cap \Phi(\Delta)=b \cap \Phi(\Delta)=\emptyset$. $f-f^{\prime}-f^{\prime \prime}$ is one-to-one and takes only values outside $\operatorname{Ran}\left(f^{\prime}\right)$ (which is the same as $\left.\operatorname{Ran}\left(f^{\prime \prime}\right)\right) . P=\bigcup\left\{P_{t}: t<\omega\right\}$. We make $p \leq p^{\prime}, p^{\prime \prime}$ and the ordering on $P$ is the one generated by this.

Lemma 2. $(P, \leq)$ is $c c c$.

Proof. Assume that $p_{\alpha} \in P\left(\alpha<\omega_{1}\right)$. We can assume by thinning and using the $\Delta$ system lemma and the pigeonhole principle that the following hold. $p_{\alpha} \in P_{t}$ for the same $t<\omega \cdot p_{\alpha}=\left(\Delta \cup a_{\alpha}, f_{\alpha}\right)$ where the structures $\left(\Delta \cup a_{\alpha},<, P\left(\Delta \cup a_{\alpha}\right), f_{\alpha}\right)$ and $\left(\Delta \cup a_{\beta},<, P\left(\Delta \cup a_{\beta}\right), f_{\beta}\right)$ are isomorphic for $\alpha, \beta<\omega_{1},\left\{\Delta, a_{\alpha}: \alpha<\omega_{1}\right\}$ pairwise disjoint. We can also assume that if $\pi$ is the isomorphism between $\left(\Delta \cup a_{\alpha},<, f_{\alpha}\right)$ and $\left(\Delta \cup a_{\beta},<, f_{\beta}\right)$ then $H$ and $\pi[H]$ are isomorphic for $H \subseteq \Delta \cup a_{\alpha}$. Moreover, if we assume that $\Delta$ occupies the same positions in the ordered sets $\Delta \cup a_{\alpha}\left(\alpha<\omega_{1}\right)$ then $\pi$ will be the identity on $\Delta$. As $\Phi(\Delta)$ is countable, by removing countably many indices we can also assume that $\Phi(\Delta) \cap a_{\alpha}=\emptyset$ for $\alpha<\omega_{1}$. Now any $p_{\alpha}$ and $p_{\beta}$ are compatible, as we can take $p=\left(\Delta \cup a_{\alpha} \cup a_{\beta}, f\right) \leq p_{\alpha}, p_{\beta}$ where $f \supseteq f_{\alpha}, f_{\beta}$ is an appropriate extension, i.e., $f-f_{\alpha}-f_{\beta}$ is one-to-one and takes values outside $\operatorname{Ran}\left(f_{\alpha}\right)$.

Lemma 3. If $(s, f) \in P$ and $H_{0}, H_{1} \subseteq s$ have $f\left(H_{0}\right)=f\left(H_{1}\right)$, then $H_{0}, H_{1}$ are isomorphic. 
Proof. Set $(s, f) \in P_{t}$. We prove the statement by induction on $t$. There is nothing to prove for $t<2$. Assume now that $(s, f) \in P_{t+1}, s=\Delta \cup a \cup b, \pi: \Delta \cup a \rightarrow \Delta \cup b$ as in the definition of $(P, \leq)$. As $f\left(H_{0}\right)$ is a value taken twice by $f$, both $H_{0}$ and $H_{1}$ must be subsets of either $\Delta \cup a$ or $\Delta \cup b$. We are done by induction unless $H_{0} \subseteq \Delta \cup a$ and $H_{1} \subseteq \Delta \cup b$ (or vice versa). Now $H_{0}$ and $\pi\left[H_{0}\right]$ are isomorphic and $f\left(H_{0}\right)=f\left(\pi\left[H_{0}\right]\right)=f\left(H_{1}\right)$, so by the inductive hypothesis $\pi\left[H_{0}\right]$ and $H_{1}$ are ismorphic, and then so are $H_{0}, H_{1}$.

Lemma 4. If $(s, f) \in P, H_{0}, H_{1} \subseteq s, f\left(H_{0}\right)=f\left(H_{1}\right), x \in H_{0} \cap H_{1}$, then $x$ occupies the same position in the ordered sets $H_{0}, H_{1}$.

Proof. Similarly to the proof of the previous lemma, by induction on $t$, for $(s, f) \in$ $P_{t}$. With similar steps, we can assume that $(s, f)=(\Delta \cup a \cup b, f) \leq\left(\Delta \cup a, f^{\prime}\right),(\Delta \cup$ $\left.b, f^{\prime \prime}\right), H_{0} \subseteq \Delta \cup a, H_{1} \subseteq \Delta \cup b$. Notice that $x \in \Delta$. Now, as $\pi(x)=x, x$ is a common element of $\pi\left[H_{0}\right]$ and $H_{1}$, and also $f^{\prime \prime}\left(\pi\left[H_{0}\right]\right)=f^{\prime \prime}\left(H_{1}\right)$. By induction we get that $x$ occupies the same position in $\pi\left[H_{0}\right]$ and $H_{1}$, so by pulling back we get that this is true for $H_{0}$ and $H_{1}$.

Lemma 5. If $(s, f) \in P, A \subseteq s, 0 \leq j \leq n$, then $A$ can be written at most $2^{j}-1$ ways as $A=H_{0} \cup H_{1}$ with $H_{0}, H_{1}$ distinct, $f\left(H_{0}\right)=f\left(H_{1}\right)$, and $\left|\gamma\left(H_{0}\right) \cap \gamma\left(H_{1}\right)\right| \geq$ $n-j$.

Proof. By induction on $j$ and, inside that induction, by induction on $t$, for $(s, f) \in$ $P_{t}$. The case $t<2$ will always be trivial.

Assume first that $j=0$. In this case our lemma reduces to the following statement. There are no $H_{0} \neq H_{1}$ such that $f\left(H_{0}\right)=f\left(H_{1}\right)$ and $\gamma\left(H_{0}\right)=\gamma\left(H_{1}\right)$. In the inductive argument we assume as usual that $s=\Delta \cup a \cup b$ and so $(s, f) \in P_{t+1}$ was created from $\left(\Delta \cup a, f^{\prime}\right)$ and $\left(\Delta \cup b, f^{\prime \prime}\right), H_{0} \subseteq \Delta \cup a, H_{1} \subseteq \Delta \cup b$. As $\gamma\left(H_{0}\right)=\gamma\left(H_{1}\right)$, $\gamma\left(H_{0}\right) \subseteq \Delta$, but then, as $\Phi(\Delta) \cap a=\emptyset, H_{0}$ can have no points outside $\Delta$ and similarly for $H_{1}$, so we can go back, say to $\left(\Delta \cup a, f^{\prime}\right) \in P_{t}$, which concludes the argument.

Assume now that the statement is proved for $j$ and we have $p=(s, f) \in P_{t+1}$, $s=\Delta \cup a \cup b$ and $p$ was created from $p^{\prime}=\left(\Delta \cup a, f^{\prime}\right)$ and $p^{\prime \prime}=\left(\Delta \cup b, f^{\prime \prime}\right)$. In $A \subseteq \Delta \cup a \cup b$ we can assume that $y=A \cap a \neq \emptyset, z=A \cap b \neq \emptyset$, as otherwise we can pull back to $p^{\prime}$ or $p^{\prime \prime}$. But then, if $A=H_{0} \cup H_{1}$, then, if, say, $H_{0} \subseteq \Delta \cup a$, $H_{1} \subseteq \Delta \cup b$ hold, then necessarily $H_{0} \cap a=y, H_{1} \cap b=z$, so $H_{0}=x_{0} \cup y$, $H_{1}=x_{1} \cup z$ where $x_{0} \cup x_{1}=x=A \cap \Delta$. We can create decompositions of $B=x \cup \pi[y] \cup z$ by taking $B=\pi\left[H_{0}\right] \cup H_{1}$. But some of these decompositions will not be different and it may happen that we get a non-proper (i.e., one-piece) decomposition. This can only happen if $\pi[y]=z$, and then the two decompositions $A=\left(x_{0} \cup y\right) \cup\left(x_{1} \cup z\right)$ and $A=\left(x_{1} \cup y\right) \cup\left(x_{0} \cup y\right)$ produce the same decomposition of $B$, namely, $B=\left(x_{0} \cup z\right) \cup\left(x_{1} \cup z\right)$, and there is but one decomposition, $A=$ $(x \cup y) \cup(x \cup z)$, which cannot be mapped to a decomposition of $B$. If this (i.e., $\pi[y]=z)$ does not happen, we are done by induction. If this does happen, we know that $\gamma\left(H_{0}\right)=\gamma\left(x_{0} \cup y\right)$ has an element in $y$ (by the argument at the beginning of the proof). As $f\left(x_{0} \cup y\right)=f\left(x_{1} \cup z\right)$, by Lemmas 3 and 4 , both $H_{0}=x_{0} \cup y$ and $H_{1}=x_{1} \cup z$ have an element in the $\gamma$-subset, at the same positions which are mapped onto each other by $\pi$. We get that $\gamma\left(x_{0} \cup z\right) \cap \gamma\left(x_{1} \cup z\right)$ has at least $n-j$ elements, so by our inductive assumption we have at most $2^{j}-1$ decompositions, which gives at most $2 \cdot\left(2^{j}-1\right)+1=2^{j+1}-1$ decompositions of $A$. 
Let $G \subseteq P$ be a generic subset. Set

$$
S=\bigcup\{s:(s, f) \in G\}, \quad F=\bigcup\{f:(s, f) \in G\} .
$$

Lemma 6. There is a $p \in P$ such that $p \|-|S|=\aleph_{n}$.

Proof. Otherwise $1 \|-\sup (S)<\omega_{n}$. By ccc, there is an ordinal $\xi<\omega_{n}$ for which $1 \|-\sup (S)<\xi$, but this is impossible as there are conditions in $P_{1}$ forcing that $\xi \in S$.

Now we can conclude the proof of the theorem. If $G$ is generic, and $p \in G$ with the condition $p$ of Lemma 6 , then in $V[G] F$ witnesses the theorem by Lemma 5 (for $j=n$ ) on the ground set $S$. As $|S|=\omega_{n}$ we can replace it by $\omega_{n}$.

\section{The GCH RESUlT}

Set

$$
T_{n}=\frac{1}{2} \sum_{i=1}^{n}\left(\begin{array}{c}
n+i \\
n
\end{array}\right)\left(\begin{array}{l}
n \\
i
\end{array}\right) .
$$

So $T_{1}=1, T_{2}=6, T_{3}=31$. In general, $T_{n}$ is asymptotically $c(3+2 \sqrt{2})^{n} / \sqrt{n}$ for some $c$.

Theorem $2(\mathrm{GCH})$. If $F:\left[\omega_{n}\right]^{<\omega} \rightarrow \omega$, then some $A \in\left[\omega_{n}\right]^{<\omega}$ has at least $T_{n}$ decompositions as $A=H_{0} \cup H_{1}, H_{0} \neq H_{1}, F\left(H_{0}\right)=F\left(H_{1}\right)$.

Proof. By the Erdös-Rado theorem (see [2], [3]) there is a set $\left\{x_{\alpha}: \alpha<\omega_{1}\right\}$ which is $(n-1)$-end-homogeneous, i.e., for some $g:\left[\omega_{1}\right]^{<\omega} \rightarrow \omega$, if $\alpha_{1}<\cdots<\alpha_{k}<\beta_{1}<$ $\cdots<\beta_{n-1}<\omega_{1}$ then

$$
f\left(\left\{x_{\alpha_{1}}, \ldots, x_{\alpha_{k}}, x_{\beta_{1}}, \ldots, x_{\beta_{n-1}}\right\}\right)=g\left(\alpha_{1}, \ldots, \alpha_{k}\right) .
$$

Select $S_{1} \in\left[\omega_{1}\right]^{\omega_{1}}$ in such a way that $g(\alpha)=c_{0}$ for $\alpha \in S_{1}$. Set $\gamma_{1}=\min \left(S_{1}\right)$. In general, if $\gamma_{i}, S_{i}$ are given $(1 \leq i<n)$, pick $S_{i+1} \in\left[S_{i}-\left(\gamma_{i}+1\right)\right]^{\omega_{1}}$ so that $g\left(\gamma_{1}, \ldots, \gamma_{i}, \alpha\right)=c_{i}$ for $\alpha \in S_{i+1}$, and set $\gamma_{i+1}=\min \left(S_{i+1}\right)$. Given $\gamma_{1}, \ldots, \gamma_{n}$ and $S_{n}$, let $\gamma_{n+1}, \ldots, \gamma_{2 n}$ be the $n$ least elements of $S_{n}-\left(\gamma_{n}+1\right)$.

Our set will be $A=\left\{x_{\gamma_{1}}, \ldots, x_{\gamma_{2 n}}\right\}$. For $0 \leq i<n$ the color of any $(n+i)$ element subset of $A$ containing $x_{\gamma_{1}}, \ldots, x_{\gamma_{i}}$ will be $c_{i}$. We can select $\frac{1}{2}\left(\begin{array}{c}2 n-i \\ n\end{array}\right)\left(\begin{array}{c}n \\ i\end{array}\right)$ different pairs of those sets which cover $A$. In toto, we get $T_{n}$ decompositions of $A$.

\section{REFERENCES}

1. J. E. Baumgartner and S. Shelah, Remarks on superatomic Boolean algebras, Annals of Pure and Applied Logic 33 (1987), 109-129. MR 88d:03100

2. P. Erdős, A. Hajnal, A. Máté, and R. Rado, Combinatorial Set Theory: Partition Relations for Cardinals, Studies in Logic, 106, North-Holland, 1984. MR 87j:04002

3. P. Erdős and R. Rado, A partition calculus in set theory, Bull. Amer. Math. Soc. 62 (1956), 427-489. MR 18:458a

4. M. Gilchrist and S. Shelah, On identities of colorings of pairs for $\aleph_{n}$ (to appear).

5. P. Komjáth, A set mapping with no infinite free subsets, Journal of Symbolic Logic 56 (1991), 1400-1402. MR 92i:03054 
6. P. Komjáth and S. Shelah, On uniformly antisymmetric functions, Real Analysis Exchange 19 (1993-1994), 218-225. MR 95b:26001

7. S. Shelah and L. Stanley, A theorem and some consistency results in partition calculus, Annals of Pure and Applied Logic 36 (1987), 119-152. MR 89d:03045

Department of Computer Science, Eötvös University, Budapest, Múzeum krt. 6-8, 1088, Hungary

E-mail address: kope@cs.elte.hu

Institute of Mathematics, Hebrew University, Givat Ram, 91904, Jerusalem, Israel

E-mail address: shelah@math.huji.ac.il 